\title{
N-Doped Graphenelike Nanostructures from $p$-Nitro Aniline-Based Foam: Formation, Structure, and Applications as a Nanofiller
}

Santosh K. Tiwari,* Ding Chen, Yu Chen, Kunyapat Thummavichai, Oluwafunmilola Ola, Zhiyuan Ma, Guangsheng Liu, Nannan Wang,* and Yanqiu Zhu

Cite This: https://doi.org/10.1021/acsomega.1c05139

Read Online

ACCESS |

山ll Metrics \& More

回 Article Recommendations

Supporting Information

ABSTRACT: Production of snake foam based on $p$-nitro aniline (PNA) was considered fun in old-school chemistry laboratories. Herein, we report the fabrication of a new carbon nanomaterial from PNA-based foam. The resulting material, resembling graphene and consisting of nitrogen heteroatoms, is $\mathrm{N}$-doped graphenelike nanostructures, and their morphology, structure, and stability are comprehensively examined using combined techniques including C-13 NMR spectroscopy, X-ray photoelectron spectroscopy (XPS), and X-ray diffraction (XRD). An optimized route was also established for their large-scale production. Further experimental validation of them as a nanofiller in polymer [SEBS (20 wt \%) and paraffin wax (80 wt \%)]-based nanocomposites was carried out, and we found that the thermomechanical properties of the nanocomposites were synchronously improved, which was attributed to the enshrouding effect of the nanofiller to the polymer chains. Owing to their good thermomechanical property and low-cost

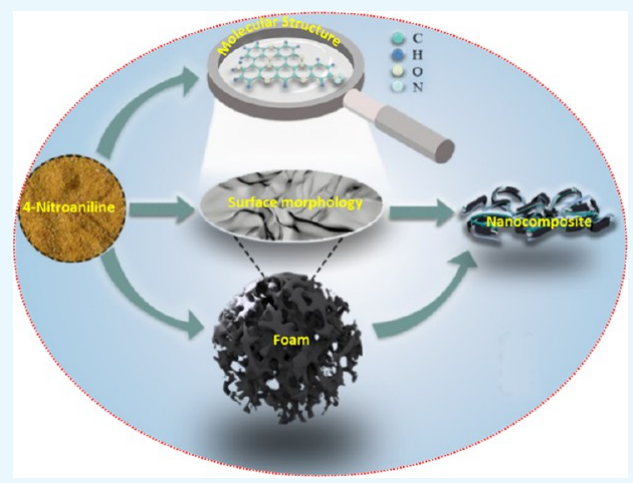
feature, these new nanomaterials can be further explored as a promising candidate for applications in energy storage, catalysis, and $\mathrm{CO}_{2}$ capture.

\section{INTRODUCTION}

Carbon is really unique, and it surprises scientific communities time and time again from the early benzene rings and complex polymers to the all-carbon caged fullerenes and tubulous nanotubes and even to the current wonder material twodimensional (2D) graphene. ${ }^{1,2}$ The diverse properties of carbon virtually exist in every phase of matter, and carbon is directly associated with many aspects of our daily life. ${ }^{2}$ Since the boom in nanomaterials in the last 2-3 decades, about 65$70 \%$ of all research outputs in materials science and engineering are directly or indirectly linked with carbon. ${ }^{3}$ In this regard, graphene and its $2 \mathrm{D}$ analogous nanocarbons have recently caught extensive research attention, due to their exceptional potentials for various engineering applications. ${ }^{4}$ While these $2 \mathrm{D}$ materials are of importance, their threedimensional (3D) form is even more promising for special applications, such as in filtration, as catalyst support, supercapacitor/battery electrodes, and as a nanofiller in fabricating high-performance composites. ${ }^{5}$ Therefore, finding a cost-effective way to create high-quality $2 \mathrm{D}$ and $3 \mathrm{D}$ graphene and its analogues and further exploring their properties are of importance. 5

Compared with their pure nanocarbon forms, $\mathrm{N}$-containing $2 \mathrm{D}$ and $3 \mathrm{D}$ graphene and graphenelike materials are more attractive, as the $\mathrm{N}$ involvement can allow for tuning the electronic, catalytic, and mechanical properties of the materials. ${ }^{6}$ Existing techniques to fabricate $\mathrm{N}$-containing graphenelike structures require several tedious steps involving the use of hazardous chemicals such as hydrazine and $\mathrm{NH}_{3}$. Low-cost polymers as raw material have been pyrolyzed for the fabrication of 3D graphene, and the pyrolysis of $\mathrm{N}$-containing polymer led to $\mathrm{N}$-containing graphenelike structures. ${ }^{8}$ Akin to melamine being used as a precursor for $\mathrm{N}$-doped carbon nanotube growth, proper $\mathrm{N}$-containing compounds could be used to synthesize $3 \mathrm{D}$ graphene foams with a certain level of nitrogen element. ${ }^{9}$ However, the key is to identify an appropriate, low-cost, $\mathrm{N}$-containing polymeric precursor that is suitable for easy processing.

Among various organic materials, aphrogen was intensively studied during the $60 \mathrm{~s}$ and $70 \mathrm{~s}$, and it has contributed largely to modern technologies. ${ }^{10}$ Aphrogen can easily form a sponge or foamlike materials upon heating ${ }^{10,11}$ and thus has been used to quench fires and improve the thermal stability of materials. ${ }^{12}$ For different aphrogens, the $p$-nitro aniline (PNA)-based foam has special attraction because it is rich in $\mathrm{N}$, similar to the $\mathrm{N}$ doped synthetic materials. ${ }^{13,14}$ Poshkus et al. first comprehensively investigated the composition, thermal stability, and

Received: September 15, 2021

Accepted: December 29, 2021 

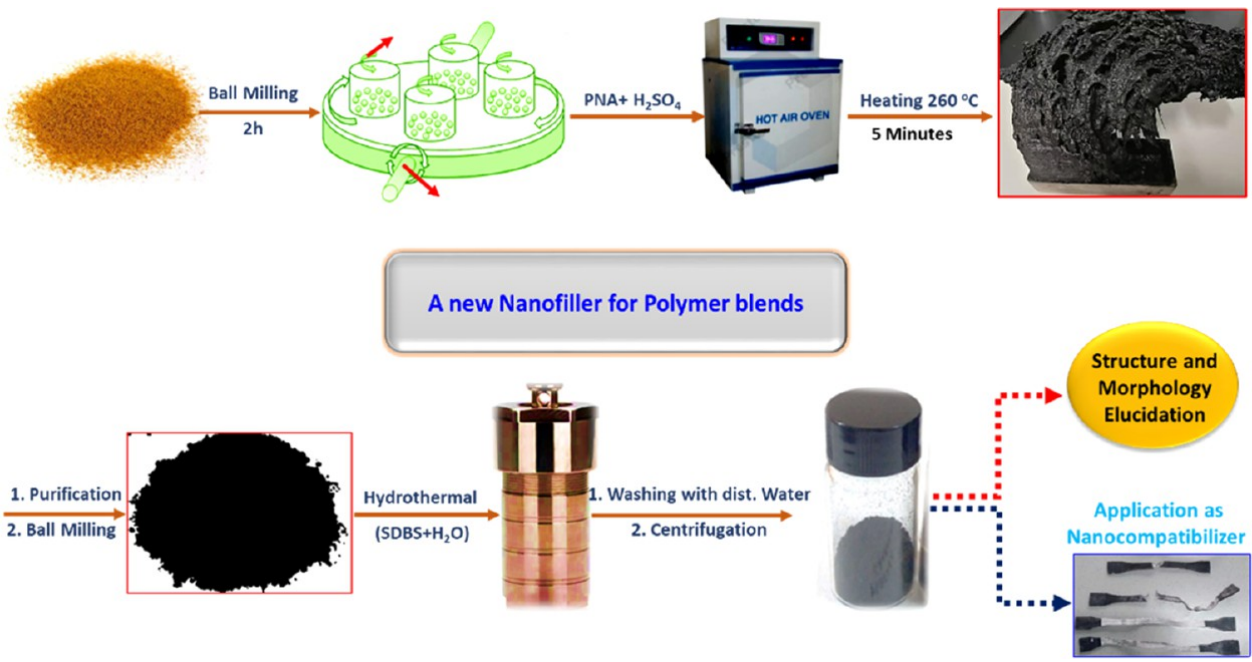

Figure 1. Schematic flowchart of PNA-based foam production, nanomaterial extraction, and their application as a nanofiller in the composites.
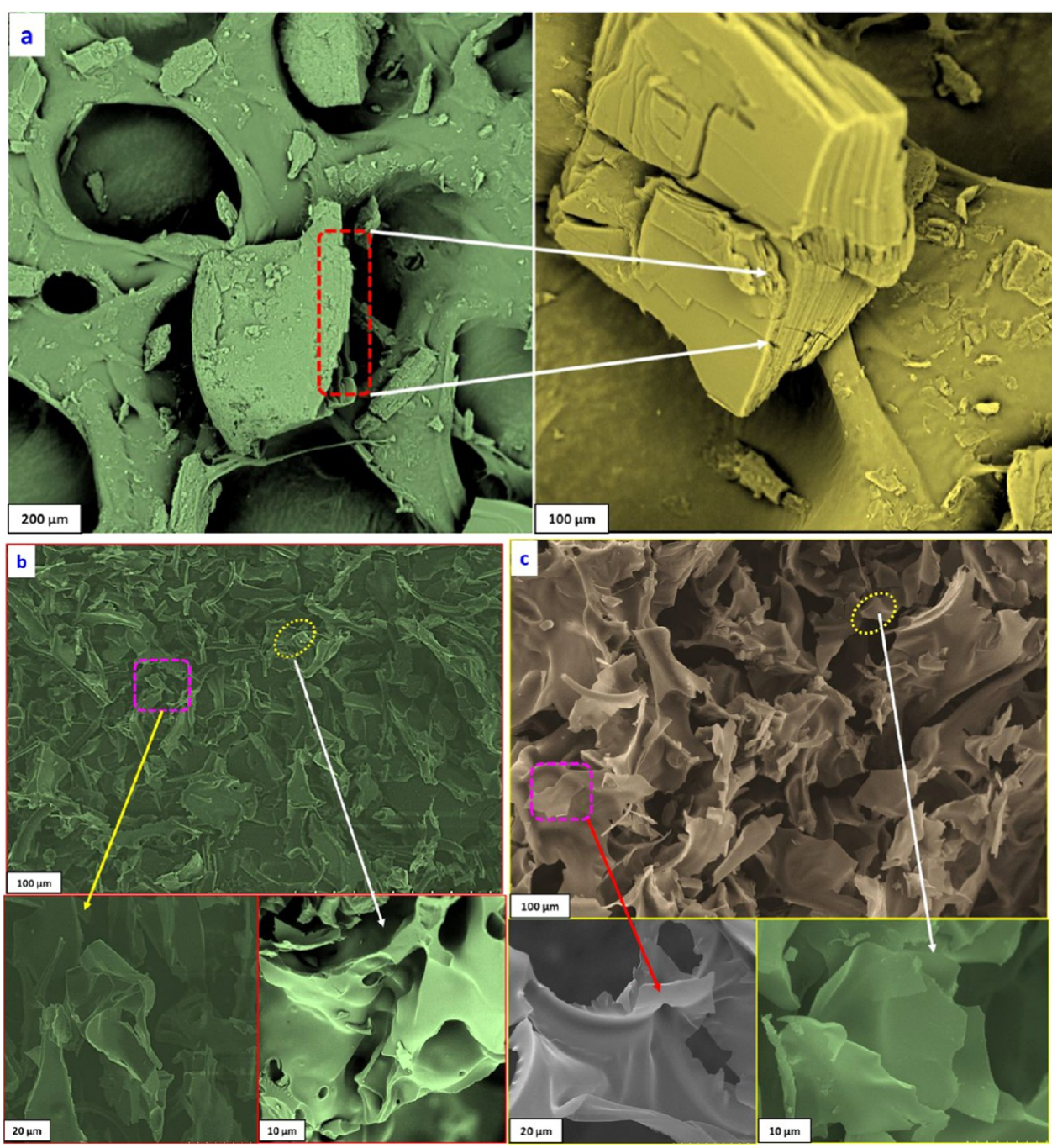

Figure 2. SEM images. (a) Pure PNA, (b) PNA-based foam, and (c) nanomaterials derived from the PNA-based foam.

features of the foam, using thermogravimetric analysis (TGA). ${ }^{10}$ They found that PNA converted to phenazine-type compounds under acidic conditions. ${ }^{10,12}$ Errede et al. further studied the structures of similar foams obtained by heating PNA at $220{ }^{\circ} \mathrm{C}$ in concentrated $\mathrm{H}_{2} \mathrm{SO}_{4},{ }^{1313}$ and they suggested that the richness in functionalities of $\mathrm{H}_{2} \mathrm{SO}_{4}$-expanded carbon foams could be used to replace the $\mathrm{KOH}$-derived activated carbon in various applications. Indeed, Andreoli et al. reported their impressive performance for utilization in $\mathrm{CO}_{2}$ adsorption. ${ }^{14}$ However, research conducted so far on PNA-based 


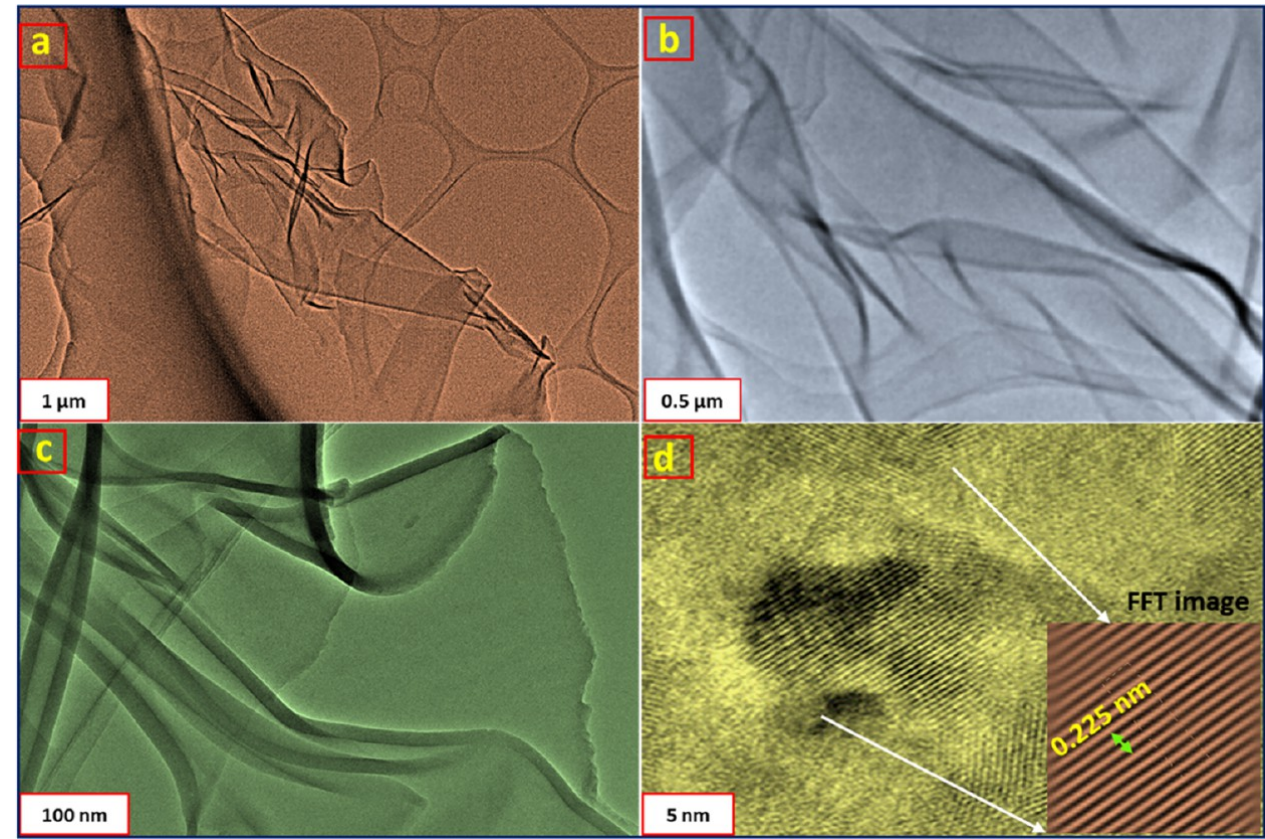

Figure 3. TEM images showing the morphologies of the PNA-based foam-extracted nanomaterials and revealing the graphenelike feature. (a-d) Images obtained at different magnifications. The inset FFT image in (d) is obtained using the Gatan Microscopy Suite Software.

foams remains on the bulk level (i.e., graphite level) and has yet to come down to the nanoregime (i.e., graphene level). This inspires us to carry out systematic studies on this intriguing material and explore the possibilities of their use in the fabrication of new materials.

Phase-change polymer materials (PCMs) were studied for thermal energy storage owing to their high storage density. ${ }^{15}$ Among numerous PCMs, paraffin is most extensively utilized for latent heat thermal energy storage. ${ }^{16}$ However, paraffinbased PCMs suffer from poor thermal stability and leakage issues during heating, ${ }^{17}$ which was tackled by mixing with SEBS in the presence of graphitic fillers. ${ }^{18}$ Considering the potential property gains of the new $\mathrm{N}$-containing graphenelike structures, we choose to validate their performance as a filler in these paraffin-based PCMs. The process flow for the PNA foam synthesis and the graphenelike material extraction is schematically presented in Figure 1.

We herein first report the optimal synthesis and characterization of new $\mathrm{N}$-doped graphenelike nanostructures from the PNA-based foam, then investigate their formation mechanism, and finally demonstrate that the resulting new materials are an effective nanofiller for SEBS and paraffin-based PCMs to achieve excellent thermomechanical stability for the composites.

\section{RESULTS AND DISCUSSION OF THE NANOMATERIALS}

2.1. SEM Analysis. As a commanding technique, scanning electron microscopy (SEM) has been widely used to portray new materials, particularly the horizon of layered nanomaterials and condensed matter. ${ }^{20}$ The extrinsic morphology of pure PNA, PNA-based foam, and new nanomaterials is presented in Figure 2. The SEM micrographs show that pure PNA at low magnification appears as particles, while at slightly higher magnifications, a huge number of sheets are stacked together, similar to the graphene layers in graphite (Figure 2). ${ }^{20,21}$ The SEM images of PNA-based foam show a belt-type morphology, along with flakes. However, the high-resolution images confirm the high thickness of the sample and very little separation of layers, and even the separated layers from the PNA-based foam do not appear to exhibit large surface areas. The SEM images (Figure 2) of the as-synthesized materials revealed that they comprise randomly gathered, thin, wrinkled sheets thoroughly linked with each other and create a disordered layered structure. $^{21,22}$ In the high-resolution SEM image, highly crumpled areas of the sheets were estimated to have an average width of 5-7 nm (as explained in Section 2.2). Interestingly, the morphology of the nanomaterials derived from the foam is very homogenous, and numerous folded sheets can be observed even at low resolution. The fieldemission scanning electron microscopy (FESEM) images of PNA-based foam show a few thick sheets of foam at $20 \mu \mathrm{m}$ magnification, while FESEM images of the nanomaterials show the morphology of several interconnected graphene oxides with a size of $10 \mu \mathrm{m}$. At the performance limit of our instrument, the images suggest the occurrence of several sheets in the nanomaterial samples, which is yet to be proved. The existence of charge during FESEM imaging also specifies that the sheets of new nanomaterials are not electrically very conductive.

2.2. TEM Analysis. We used TEM to examine the nature of the layers, thickness, and $d$-spacing of the derived nanomaterials, and the results are presented in Figure 3 (the TEM image of native PNA foam is depicted in Figure S1 in the SI). The few-layer feature of the sample was easily visible even at low magnification, Figure $3 a, b$, and the contrast and brightness of images were highly consistent throughout, which implies the uniform thickness of the sheets. ${ }^{23}$ Large nanosheets on a $\mathrm{Cu}$ gird were pragmatic, smooth, and wavy, akin to the morphology of the pristine few-layer graphene. ${ }^{23}$ Moreover, the nanosheets are quite transparent, being stable under irradiation of the electron beam. From the high-resolution transmission electron microscopy (HRTEM) images in Figure $3 \mathrm{c}, \mathrm{d}$, the nanosheets were continuously folded back (Figure 
3c) so that the exposed boundaries and edges allowed for a cross-sectional examination to count the number of layers and measure the layer space. We found a sporadic layer, which was attributed to the presence of amorphous carbon in the nanosheets. Some well-defined lattices in Figure 3d indicated a certain degree of crystallinity in the materials. The distance between atomic planes was measured to be $0.225 \mathrm{~nm}$, quite close to that reported for the graphenelike structure containing nitrogen heteroatoms. ${ }^{24,25}$ Using $\mathrm{N}_{2}$ gas absorption, we obtained a Brunauer-Emmett-Teller (BET) specific surface area value of $\sim 83 \mathrm{~m}^{2} / \mathrm{g}$ (Figure $\mathrm{S} 2$ in the SI) for the resulting nanosheets, which indicates a moderate degree of exfoliation of sheets from PNA.

2.3. FTIR Analysis. To observe the functional groups in pure PNA, PNA-based foam, and nanosheets extracted from the PNA foam, Fourier-transform infrared (FTIR) spectroscopy analyses were carried out in attenuated total reflection (ATR) mode. Pure PNA showed (Figure 4a) characteristic

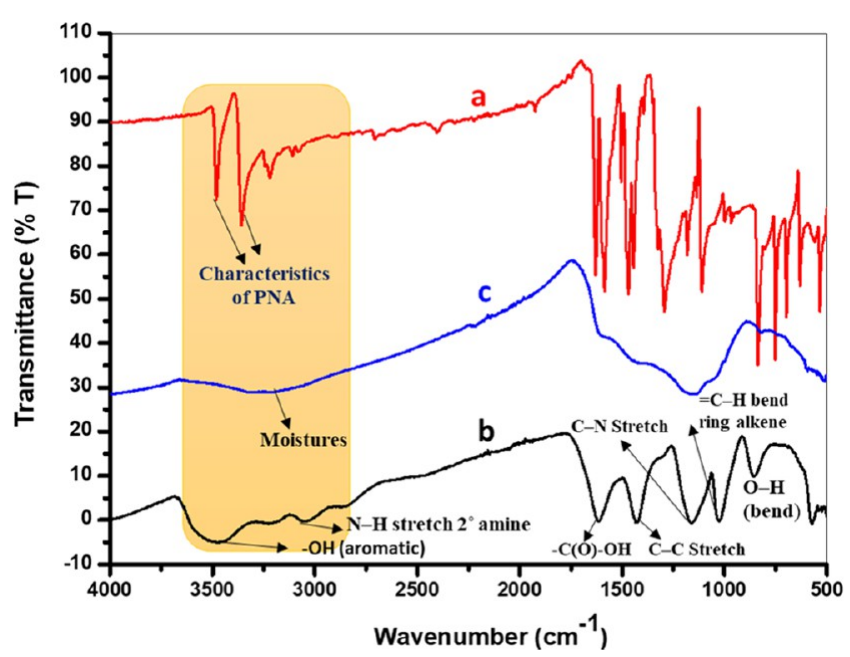

Figure 4. AT-FTIR spectra of (a) pure PNA, (b) PNA-based foam, and (c) extracted nanomaterials from PNA-based foam.

functional groups, including $-\mathrm{NH}_{2}, \mathrm{NO}_{2},-\mathrm{C}=\mathrm{C}-,-\mathrm{N}-\mathrm{C}$, etc., in line with the reference. ${ }^{25}$ The as-produced PNAderived foam consists of $\mathrm{N}$-, $\mathrm{C}$-, and $\mathrm{O}$-based functional groups, as marked in Figure 4. A broad absorption peak at around $3212 \mathrm{~cm}^{-1}$ is visible, arising from the $\mathrm{N}-\mathrm{H}$ stretching of secondary amines, along with the $\mathrm{C}-\mathrm{C}$ aromatic stretching. The $\mathrm{C}-\mathrm{C}$ stretch at $1601 \mathrm{~cm}^{-1}$ (in-ring) is quite broad too, implying fewer functional units in the sample. Infrared (IR) spectroscopy absorption at $\sim 1020 \mathrm{~cm}^{-1}$, matching $=\mathrm{C}-\mathrm{H}$, offers strong evidence for the unconjugated alkene in the PNAbased foam (Figure 4b) but not in the extracted nanomaterials (Figure 4c). A clear $\mathrm{C}-\mathrm{N}$ stretch peak at $1176 \mathrm{~cm}^{-1}$ implies that $\mathrm{N}$ atoms exist mostly in the ring for both cases. The absence of peaks at $\sim 3448,3312$, and $\sim 856 \mathrm{~cm}^{-1}$ in the extracted new materials is proof that they contain no such functional groups on the surface. Therefore, $\mathrm{N}$ atoms are likely to locate within the ring systems, as reported in the case of $\mathrm{N}$ doped graphene and graphene oxide. ${ }^{26}$ The FTIR peaks for the extracted nanomaterials appear to be fewer in the range of $1500-800 \mathrm{~cm}^{-1}$, indicating that few carbon atoms are in the aliphatic rings as well. The $-\mathrm{H}-\mathrm{O}$ bending peak is also observed, which is possibly attributed to moisture. Based on the FTIR results, the difference between the newly derived nanomaterials and either pure PNA or the PNA-based foam is quite clear. Further, detailed information about the functional groups and elemental composition is discussed in Section 2.4.

2.4. XPS Analysis. XPS spectra of the PNA-based foam and its derived nanomaterials are presented in Figure 5, while the spectra of pure PNA have been discussed elsewhere. ${ }^{27}$ The survey spectra confirmed a high amount of $\mathrm{N}, \mathrm{O}$, and $\mathrm{C}$ in the PNA foam; however, an ominously lesser amount of $\mathrm{O}$ in the extracted nanomaterials was observed, when compared between Figure 5a and b. Grippingly, peaks matching the graphenelike $\mathrm{N}$-doped materials were also found to be reduced, which indicates a reduced quantity of $\mathrm{N}$ functional groups in the sample. ${ }^{28}$

The deconvoluted $\mathrm{O} 1 \mathrm{~s}$ spectrum (inset of Figure $5 \mathrm{~b}$ ) shows that the $\mathrm{N}$-containing graphenelike nanomaterials derived from the PNA foam have a very less amount of $\mathrm{O}$ compared to the parental PNA foam. The $\mathrm{C}$ 1s signals of the PNA foam (Figure $5 \mathrm{a}, \mathrm{b})$ reveal both aromatic and aliphatic $\mathrm{C}$, along with a variety of $\mathrm{O}$ - and $\mathrm{N}$-based functional groups, as denoted in the case of the PNA-based foam. However, the $C$ 1s spectra of the extracted sample (inset of Figure 5b) are much more prominent than those of the PNA-based foam, possessing fewer functional units. The XPS spectra of both the PNAbased foam and its extracted nanomaterial show $\mathrm{sp}^{3}$ hybridized carbon, which is probably owing to the residue carbon encountered on surfaces prior to the analysis. Large amounts of aromatic $\mathrm{sp}^{2}$ hybridized carbon are observable in both materials, which maintain their aromaticity rings even in the doped structures. The signal of $\pi \rightarrow \pi^{*}$ transition corresponds to the aromatic ring at $\sim 291.4 \mathrm{eV}$, which is evidence for the $\mathrm{N}$ -
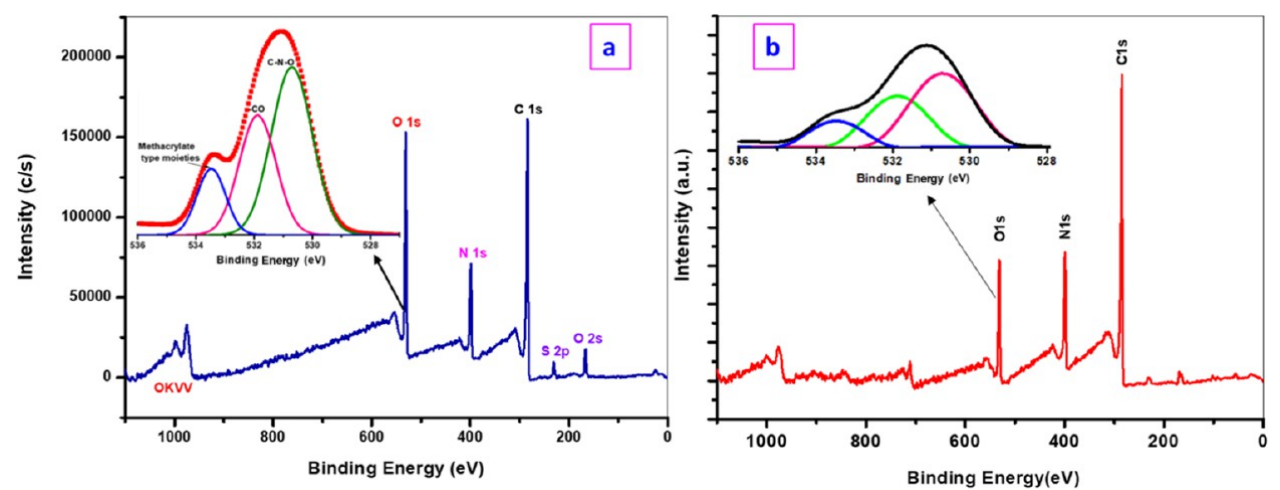

Figure 5. XPS survey spectra of (a) the PNA-based foam and (b) extracted N-containing nanomaterials. The insets show the deconvoluted O 1s spectrum. 
doped nanostructures in the deconvoluted C 1 s XPS peak. ${ }^{27}$ The $C$ 1s peak in the extracted nanomaterials is more intensified compared to that of the PNA foam, due to the abundance of the aromatic $\mathrm{sp}^{2}$ hybridized carbon.

A substantial amount of carbon is found at the strangely low binding energy of $283.5 \mathrm{eV}$ in the $\mathrm{N}$-doped sample. This must be associated with the chemistry involved during the expansion process. This binding energy can be assigned to conjugated $\mathrm{sp}^{2}$ carbon, which tends to appear due to the oxidized form of carbon, similar to other foaming materials. ${ }^{27,28}$ The peaks at higher binding energy for both materials are related to the carbon atoms having different functional groups, as assigned in Table 1. Thus, the states of the extracted N-containing

\section{Table 1. Summary of the Binding Energies and Their} Corresponding Assignments of the PNA-Based Foam

\begin{tabular}{|c|c|c|}
\hline s.no. & functional units in PNA-based foam & peaks $(\mathrm{BE})$ \\
\hline \multicolumn{3}{|c|}{ carbon $1 \mathrm{~s}$} \\
\hline 1 & alkene conjugated carbon & 283.5 \\
\hline 2 & aromatic carbon & 284.9 \\
\hline 3 & $\mathrm{C}-\mathrm{N}-\mathrm{C}$ & 286.4 \\
\hline 4 & $-\mathrm{C}(\mathrm{O}) \mathrm{NH}$ or $-\mathrm{C}=\mathrm{O}$ & 287.7 \\
\hline 5 & $\pi \rightarrow \pi^{*}$ transition of the aromatic ring & 291.4 \\
\hline 6 & alkene conjugated carbon & 283.5 \\
\hline 7 & aromatic carbon & 284.9 \\
\hline 8 & $\mathrm{C}-\mathrm{N}-\mathrm{C}$ & 286.4 \\
\hline \multicolumn{3}{|c|}{ oxygen $1 \mathrm{~s}$} \\
\hline 9 & methacrylate, terephthalate-type moieties & 531.8 \\
\hline 10 & $\mathrm{H}_{2} \mathrm{O},-\mathrm{CO}$ & 530.8 \\
\hline 11 & $\mathrm{C}-\mathrm{N}-\mathrm{O}$ & 530.1 \\
\hline \multicolumn{3}{|c|}{ nitrogen $1 \mathrm{~s}$} \\
\hline 12 & $\mathrm{NO}_{2},-\mathrm{N}=\mathrm{O},=\mathrm{NH}$ & 404.8 \\
\hline 13 & $N$-oxide & 303.2 \\
\hline 14 & $\mathrm{C}(\mathrm{O}) \mathrm{NH}, \mathrm{N}-\mathrm{N},-\mathrm{N}=\mathrm{C}$ & 404.3 \\
\hline 15 & $-\mathrm{NH}_{2}$, aromatic $\mathrm{N}$ & 399.1 \\
\hline
\end{tabular}

nanostructures and the PNA-based foam are quite different. The $\mathrm{C}-\mathrm{N}-\mathrm{C}$, aromatic $\mathrm{N}, \mathrm{N}-\mathrm{N}$, and $-\mathrm{N}=\mathrm{C}$ peaks of the XPS results offer strong evidence that most of the $\mathrm{N}$ atoms are indeed located in the rings. Further, C-13 NMR spectroscopy evidence is shown in Figure S3 in the SI. Based on the XPS spectra, the $\mathrm{N}$-doped nanomaterials consist of $75 \%$ of C, $15 \%$ of $\mathrm{O}$, and $10 \%$ of $\mathrm{N}$ by weight. The deconvoluted XPS spectra of pure PNA foam are presented in Figure S5 in the SI for a comparative study.
2.5. Raman Analysis. The Raman spectra of the PNAbased foam and its derived nanostructures are presented in Figure 6, while the Raman spectrum of pure PNA is discussed elsewhere. $^{29,30}$ The Raman features of the present extracted nanomaterials (Figure 6a) are similar to those of graphene containing heteroatoms. ${ }^{26}$ In contrast, the spectrum of the PNA-based foam (Figure $6 \mathrm{~b}$ ) resembles that of amorphous carbon, which contains defects and side chains as proposed previously in the literature. ${ }^{31-33}$ The extracted sample also presents D, G, and 2D Raman shifts, and they upshifted toward higher frequency (related to graphene), which distinguishes them from graphitic systems. ${ }^{34-36}$ The D and G bands in our samples are owing to the presence of polyaromatic hydrocarbon lattices dominated by $\mathrm{sp}^{2}$ sites, along with a few $\mathrm{sp}^{3}$ sites because visible excitation only resonates with the $\pi$ states. ${ }^{32,33}$ Herein, the $\mathrm{G}$ band is due to the bond stretching of all pairs of $\mathrm{sp}^{2}$ atoms in six-membered rings, while the $\mathrm{D}$ band is due to the breathing modes of $\mathrm{sp}^{2}$ atoms in rings and $\mathrm{sp}^{3}$ hybridized carbon atoms at edges. ${ }^{31-33}$ The upshifts imply the presence of $\mathrm{N}$ atoms in the ring, which cause notable distortion in the crystal lattice, as elaborated by other researchers. ${ }^{35,36}$ The intensity ratio of $D$ and $G$ bands offers a gauge for the number of essential defects and serves as a quantitative measure of the edge plane exposure. The extracted nanostructures have an $I_{\mathrm{D}} / I_{\mathrm{G}}$ ratio of $\sim 1.13$, similar to that of $\mathrm{N}$-doped graphene systems reported elsewhere. ${ }^{34,36}$ The small ratio indicates a reduction in the average size of the $\mathrm{sp}^{2}$ domains, which is due to the incorporation of $\mathrm{N}$ atoms and their covalent bonding with $\mathrm{C}$ atoms. ${ }^{37,38}$ For pristine graphene, it shows a remarkably intense 2D Raman shift, roughly four to five times more than the $\mathrm{G}$ band; ${ }^{30,32}$ however, the extracted nanomaterials only show a broad $2 \mathrm{D}$ band at around $2860 \mathrm{~cm}^{-1}$, which is dissimilar to the spectrum of the PNA-based foam itself. This broadness of the 2D band directly corresponds to the number of layers and thickness. ${ }^{31,32}$ The upshifting of the $2 \mathrm{D}$ band in the extracted sample also corresponds to its increased sheet thickness and hugely folded layers. ${ }^{35-39}$

2.6. XRD Analysis. Powder XRD patterns of the PNAbased foam and its derived nanomaterials are shown in Figure 7. We found that the pattern for pure PNA (Figure 7a) was identical to those discussed by Wang et al., ${ }^{37}$ while being different from those of the two types of $\mathrm{N}$-containing samples. The PNA-based foam (Figure $7 \mathrm{~b}$ ) shows diffraction peaks at $2 \theta=22.2,24.5,33.9$, and $37.8^{\circ}$, similar to those of the mesophase pitch-based carbon foams, ${ }^{40,41}$ while the extracted
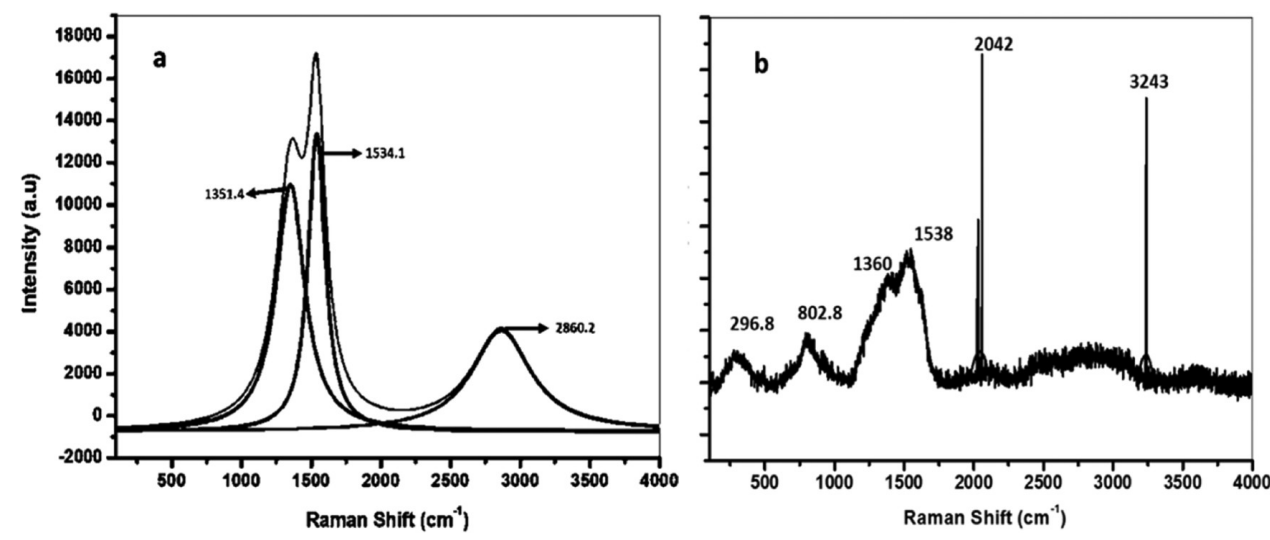

Figure 6. Raman spectra of (a) the PNA-based foam and (b) its derived nanomaterials. 


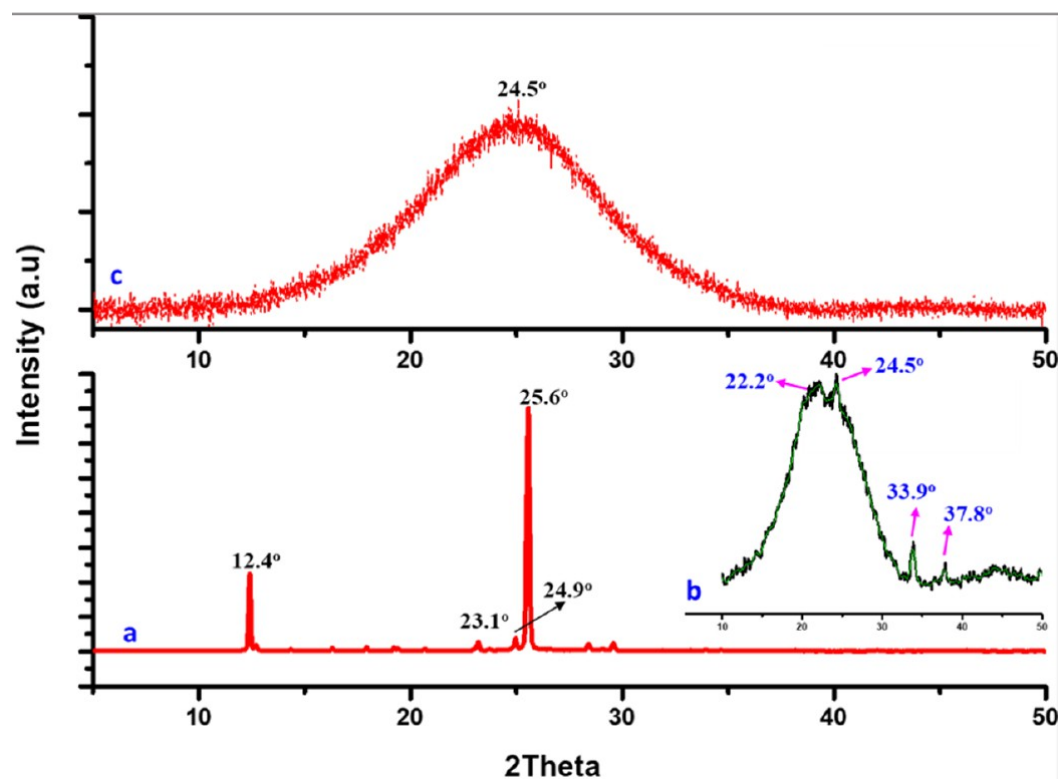

Figure 7. Powder XRD spectra. (a) Pure PNA, (b) PNA-based foam, and (c) extracted nanomaterials, which show features of N-doped graphene (plotted separately due to huge intensity differences).

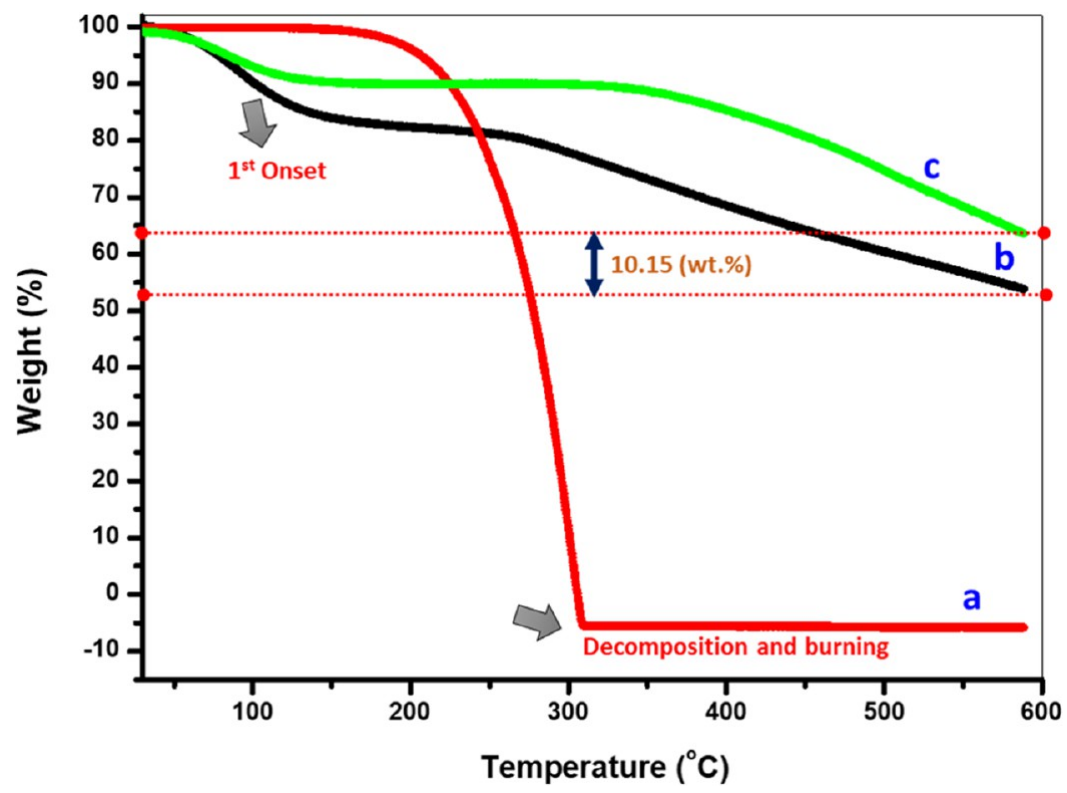

Figure 8. TGA curves. (a) Pure PNA, (b) PNA-based foam, and (c) extracted nanomaterials.

sample only shows one broad peak at $2 \theta=24.5^{\circ}$ for the (002) plane (Figure 7c), which signifies its highly disordered feature. ${ }^{37,38}$ However, compared with the PNA-based foam, this broad peak is much weaker and lacks the bifurcated feature. ${ }^{42}$ The position of the XRD band of (002) is found to slightly shift toward the lower $2 \theta$ value along with high broadness in the case of the $\mathrm{N}$-doped sample, and it is strong evidence for randomness in the crystal lattice. ${ }^{38,41}$ The common weak peak at $\sim 42.5^{\circ}$ for graphitic carbon is absent for the extracted sample, which suggests that the present nanomaterial has a layered structure but is different from that of the graphite family. ${ }^{41,42}$ This result implies that the crystallinity of the extracted sample is between the amorphous state and graphenelike crystal. This structure is formed by an arbitrary linkage of benzene units one by one during polymerization (Figure S4 in the SI) of layers in the material. ${ }^{40,41}$

2.7. Thermal Stability. The thermal stability of all three samples was evaluated by thermogravimetric analysis and TGA thermograms (at a $10{ }^{\circ} \mathrm{C} / \mathrm{min}$ heating rate under $\mathrm{Ar}$ ), and the results are displayed in Figure 8. There is no weight loss from 100 to $110{ }^{\circ} \mathrm{C}$ for PNA, confirming no water and moistures absorption in it. A rapid weight loss occurred at $161{ }^{\circ} \mathrm{C}$, originating from the removal of functional groups. Following a mild weight loss of only 5-6\% from 185 to $200{ }^{\circ} \mathrm{C}$, Figure $8 \mathrm{a}$, another rapid weight-loss stage was observed after $200{ }^{\circ} \mathrm{C}$, due to the complete decomposition of PNA. This result is in line with the literature report. ${ }^{43}$ In contrast, the PNA-based foam (Figure $8 \mathrm{~b}$ ) showed a much larger weight loss (14-16 wt \%) from 100 to $160{ }^{\circ} \mathrm{C}$, which might be due to the removal of moisture, functional groups, and small side chains. The 

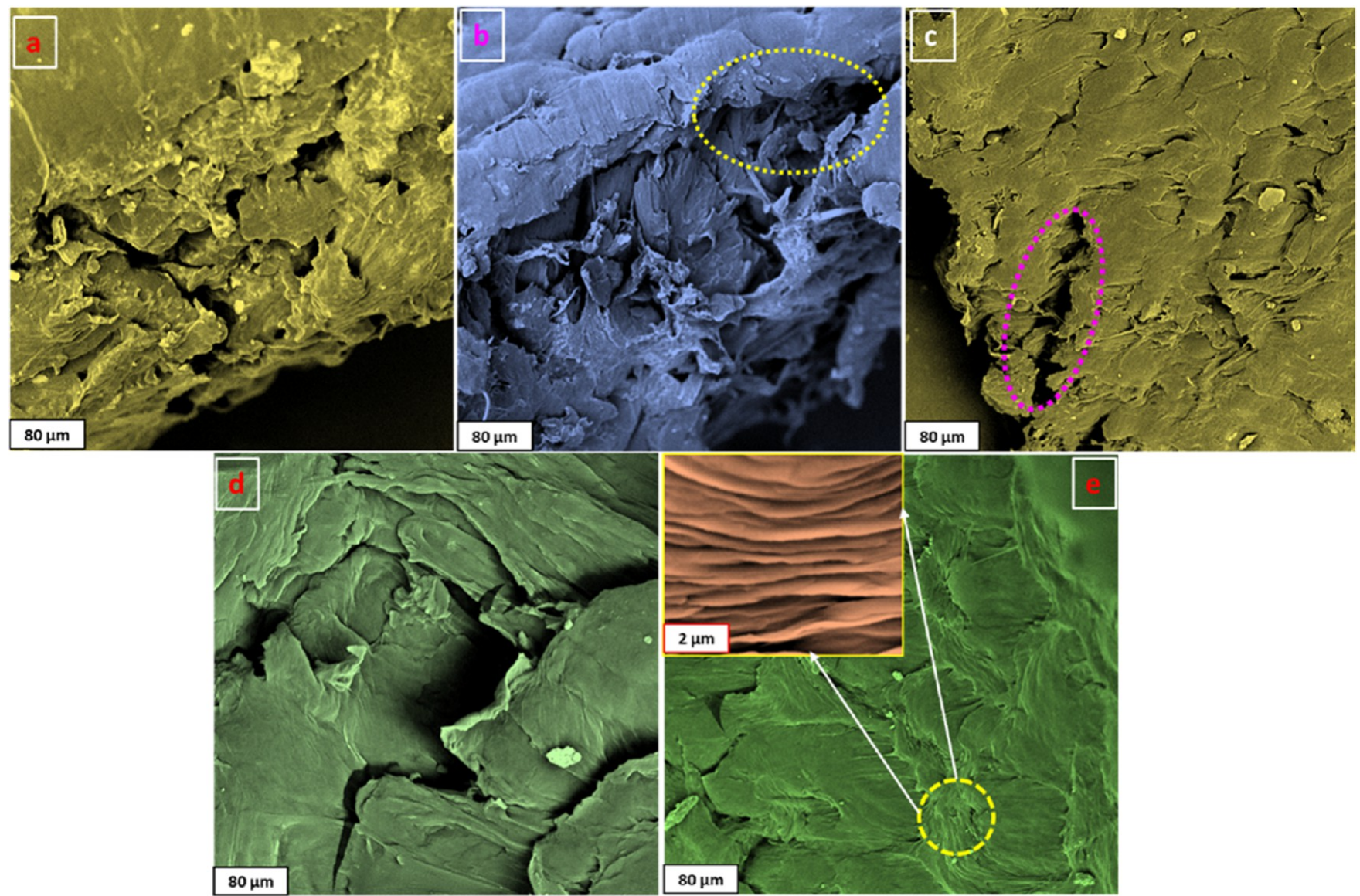

Figure 9. SEM images of the fractured surface. (a) Pure paraffin and SEBS blend, (b) PS0.25, (c) PS0.50, (d) PS0.75, and (e) PS01.

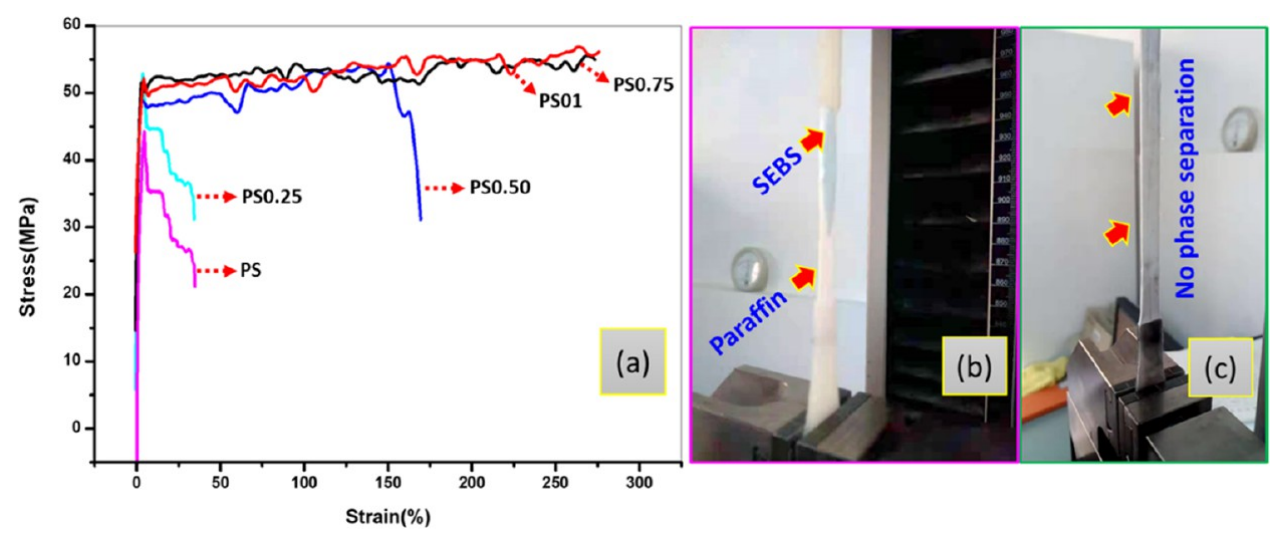

Figure 10. (a) Stress-strain curves of the pure blend and nanocomposites, (b) digital photograph of the pure blend specimen showing phase separation, and (c) digital photograph of PSO1 displaying no phase separation.

extracted sample, however, showed a much lesser weight loss across the entire temperature range, as shown in Figure 8c, and eventually showed a remaining weight of $10.15 \mathrm{wt} \%$ more than that of the PNA-based foam. This appears to suggest that it contained fewer amounts of functional groups and amorphous carbon than the PNA-based foam, which is comparable to the reduced few-layer graphene oxides. ${ }^{34,44}$ Due to the fewer amounts of $\mathrm{O}$ - and $\mathrm{N}$-functional groups on the surface and at the edges, the extracted $\mathrm{N}$-containing nanomaterials retained more than $70 \%$ of their original weight even at $600{ }^{\circ} \mathrm{C}$. These results imply that no skeletal change occurred when the $\mathrm{N}$ doped sample was heated in an inert environment. Based on our microscopic and spectroscopic investigations, we proposed the likely pathway for the conversion of $\mathrm{N}$-containing graphenelike nanomaterials from PNA, and an illustration is presented in Figure S4, SI.

\section{RESULTS AND DISCUSSION OF NANOCOMPOSITES}

3.1. FESEM Analysis. Figure 9 shows the fractural surfaces of the composite specimens after breaking in liquid nitrogen. The pure blend of paraffin and SEBS showed a lot of spacing, as shown in Figure 9a, possibly owing to the incompatibility between paraffin and SEBS. Samples PS0.25 and PS0.50 showed a similar fracture surface, while PS0.75 and PS01 
showed no significant differences under low magnification. For the low-content samples (PS0.25 and PS0.50), cracks were seen on the surface, Figure $9 a, b$, and the filler distribution was sparse, insufficient to cover the entire surface of the nanocomposites. Sample PS01, however, showed a different surface morphology; the filler distribution was uniform and covered the entire surface, and a dramatic decrease in the size of SEBS and paraffin domains became visible (Figure 9e). Compared with PSO.50 whose fracture surface was rough and full of cracks, the surface of PS01 was highly compact and appeared smooth, indicating that an ideal content level was obtained and improved mechanical properties were to be expected. Further at high magnification, inset in Figure 9e, sample PS01 showed that the layered fillers along with the crack exhibited no voids on the fracture surface, demonstrating their good compatibility with the matrix and the high quality of the composites.

3.2. Tensile and Microhardness Valuation. The stressstrain curves of the specimens are exhibited in Figure 10. The pure blend sample displayed a typical elastomeric behavior and exhibited a fracture strength of $25 \mathrm{MPa}$; in contrast, the incorporation of the new $\mathrm{N}$-containing graphenelike fillers in the blends improved the tensile strengths, as shown in Figure 10a. During testing, we observed phase separation in the pure blend and lower-filler-content samples but not in PS01, as shown in Figure 10b,c, which is owing to the incompatibility between paraffin and SEBS. Even for PSO.50, the elasticity of the filler did not transfer the load successfully to the blend matrix, although the tensile strengths were gradually increased with higher filler contents ${ }^{45,46}$ (as discussed in the SI Mechanical Properties section, Figure S11). Sample PS01 exhibited the maximum tensile strength, double that of PSO.50, owing to a sufficient amount of filler forming a homogenous coverage and tightening the polymer chains, as discussed in the FTIR, TGA, and DSC sections (Figures S7-S10) in SI. The stiffness of the composites also increased greatly along with increased filler loading (Figure 10a). We believe that the nanofiller functions as a load dispersion agent owing to its high mechanical stability and lightweight (Figure S6, SI); therefore, this minimizes crack generation and propagations, therefore improving the strength. Table 2 shows the average Vickers hardness values of the composite samples, which were recorded on a Zwick I-Z2.5 instrument, with a loading time of $12 \mathrm{~s}$.

Table 2. Hardness Values of the Pure Blend and the Nanocomposites

$\begin{array}{cccc}\text { s.no. } & \text { sample code } & d(\mu \mathrm{m}) & \text { hardness (HV 0.2) } \\ 1 & \text { PS } & 766.1 & 0.644 \\ 2 & \text { PS } 0.25 & 677.6 & 0.82 \\ 3 & \text { PS0.50 } & 652.1 & 0.88 \\ 4 & \text { PS } 0.75 & 646.7 & 0.90 \\ 5 & \text { PS01 } & 619.9 & 1.0\end{array}$

\section{CONCLUSIONS}

A layered $\mathrm{N}$-containing carbon nanostructure resembling graphene has been produced from $p$-nitro aniline-based foam. An optimized and well-defined technique for the synthesis of this $\mathrm{N}$-doped graphenelike nanostructure has been recognized, which is suitable for bulk production, and its conversion mechanism for $p$-nitro aniline-based foam production has been established. The surface characteristics of the extracted nanomaterials were comprehensively evaluated using BET, HRTEM, and FESEM analyses, and their chemical structure was determined using C-13 NMR spectroscopy, XPS, and powder XRD. We have further validated their potential applications as a filler to enhance the thermomechanical properties in paraffin-SEBS blend matrixes. At a very small amount of filler loading, the resulting composites showed improved thermomechanical properties, with a $\sim 40 \%$ enhancement in hardness and a $\sim 7 \%$ increase in thermal properties. We believe that this new $\mathrm{N}$-doped nanomaterial may also find use in electrodes, biosensors, and water purification in the near future.

\section{EXPERIMENTAL SECTION}

5.1. Materials and Equipment. Crystalline PNA (1.8-2 $\mu \mathrm{m}$ ), styrene ethylene butylene styrene (SEBS, molecular weight of $1.5 \times 10^{4}$, with polydispersity less than 1.04 , and the amount of styrene block of ca. 30\%), paraffin wax (major component is $n$-octadecane), reagent-grade sulfuric acid (98\%), ethanol, and acetone were purchased from Reagent Co., Ltd. (Shanghai) and Shell China. All of these materials and solvents were utilized without further purification. A ball mill machine (3D rotation), furnace, bath sonicator, and centrifuge machine were used for the synthesis and product purification.

5.2. Foam Production from PNA. 5.2.1. Step 1: PNA Foam Formation. Pure PNA was first ball-milled $(500 \mathrm{rpm}$, $3 \mathrm{D}$ rotation) for $2 \mathrm{~h}$, and the powder was dried for $24 \mathrm{~h}$ at 100 ${ }^{\circ} \mathrm{C}$ under vacuum. Second, the fine PNA powder was mixed with $\mathrm{H}_{2} \mathrm{SO}_{4}$ in a mortar at different molar ratios of $0.1: 1,0.2: 1$, $0.2: 1,0.3: 1,1: 1,1: 2$, and 0.3:2. Third, the mixture was heated in the $240-260{ }^{\circ} \mathrm{C}$ range for $5 \mathrm{~min}$ in a furnace, which resulted in the rapid creation of "snake-like" foams. If overheated, e.g., for 5-7 min, the sample would start burning and no nanosized materials are formed. Finally, the resulting foams were thoroughly rinsed using deionized water and ethanol to remove excess acid and unreacted PNA and dried at $100{ }^{\circ} \mathrm{C}$ in a hot air oven for $24 \mathrm{~h}$.

5.2.2. Step 2: Graphenelike Sample Extraction. The abovepurified foam was further heated at $300{ }^{\circ} \mathrm{C}$ for $2 \mathrm{~h}$, then subjected to ball milling and sonication for $1 \mathrm{~h}$ (at $500 \mathrm{rpm}$ ) and $30 \mathrm{~min}$ (in distilled water), respectively. After these treatments, large particles that remained in the sample were removed by centrifugation. To produce high-quality layered nanostructures, $0.1 \mathrm{~g}$ of sodium dodecylbenzene sulfonate (SDBS) was then added to the supernatant liquid $(100 \mathrm{~mL})$ in distilled water, which was then treated hydrothermally at 120 ${ }^{\circ} \mathrm{C}$ for $5 \mathrm{~h}$. SDBS was used as an antiblocking and surfacecleaning agent. After the hydrothermal treatment, we obtained materials with a yield of $\sim 50-55$ wt $\%$ from PNA.

5.3. Preparation of Nanocomposites. To prepare paraffin and triblock copolymer SEBS (Kraton G1624)-based nanocomposites, a solution processing method reported by Zhang et al. was used for mixing with the nanofiller. ${ }^{19}$ Briefly, 20 wt \% SEBS and 8 wt \% paraffin wax, along with 0 wt \% (PS), 0.25 wt \% (PS0.25), 0.50 wt \% (PS0.50), 0.75 wt \% (PS0.75), and 1 wt \% (PS01) nanofiller, were dissolved in 100 $\mathrm{mL}$ of cyclohexane, which was continuously magnetically stirred at $300 \mathrm{rpm}$ and $70{ }^{\circ} \mathrm{C}$ for $10 \mathrm{~h}$. Then, the mixture was further heated at $90{ }^{\circ} \mathrm{C}$ while stirring, until almost all cyclohexane was evaporated. After this, the powder mixture was dried in a vacuum chamber at $100{ }^{\circ} \mathrm{C}$ to remove any liquid 
residue and air from voids. Finally, the resulting composites were molded as dog bone specimens for further mechanical property evaluation.

5.4. Characterizations. The surface morphology, internal structure, and spectroscopic features of the resulting foams and the associated composites were examined using combined techniques, including field-emission scanning electron microscopy (FESEM, SU8020 and SEM, S-3400N), high-resolution transmission electron microscopy (HRTEM, $300 \mathrm{kV}$, Tecnai G2 F30, FEI), Raman spectroscopy (inVia Reflex, Renishaw), ultraviolet spectroscopy (UV, LAMBDA 365, Perkin Elmer Co., Ltd.), and X-ray photoelectron spectroscopy (XPS, Escalab 250XI, Thermo Fisher Scientific Ltd.). The X-ray diffraction (XRD, SMARTLAB3KW) patterns were acquired with a D/MAX2200/PC X-ray diffractometer with $\mathrm{Cu} \mathrm{K} \alpha$ radiation $(\lambda=0.154 \mathrm{~nm})$. The surface area of the resulting foams was evaluated using a Brunauer-Emmett-Teller (BET) instrument (TriStar II3020, Micromeritics Instrument Co., Ltd.). The thermal stability of samples was investigated using thermogravimetric analysis linked with a FTIR machine (TGAFTIR, TGA4000 Spectrum Two TL8000, Perkin Elmer Co., Ltd.). Thermomechanical properties were tested using TGA, differential scanning calorimetry (DSC, Netzsch 200F3, Germany), and a UTM-Hardness machine (Zwick Roell IZ2.5). Tensile testing is carried out using the dog bone specimens.

\section{ASSOCIATED CONTENT}

\section{S1 Supporting Information}

The Supporting Information is available free of charge at https://pubs.acs.org/doi/10.1021/acsomega.1c05139.

TEM analysis of PNA foam (Figure S1); BET analysis of PNA foam and nanomaterial (Figure S2); solid-state C13 NMR spectrum of nanomaterials (Figure S3); probable mechanism of $\mathrm{N}$-doped graphenelike materials from PNA (Figure S4); deconvoluted XPS spectra of PNA foam and nanomaterial (Figure S5); mechanical strength of foam and its lightness (Figure S6); FTIR analysis of nanocomposites (Figure S7); DSC analysis (Figure S8); schematic of the enshrouding effect (Figure S9); TGA analysis (Figure S10); and tensile properties of nanocomposites (Figure S11) (PDF)

\section{AUTHOR INFORMATION}

\section{Corresponding Authors}

Santosh K. Tiwari - Guangxi Institute Fullerene Technology (GIFT), Key Laboratory of New Processing Technology for Nonferrous Metals and Materials, Ministry of Education, School of Resources, Environment and Materials, Guangxi University, Nanning 530000, China; Department of Chemistry, Warsaw University, Warsaw 00-927, Poland; ○ orcid.org/0000-0003-1602-9345; Email: ismgraphene@ gmail.com

Nannan Wang - Guangxi Institute Fullerene Technology (GIFT), Key Laboratory of New Processing Technology for Nonferrous Metals and Materials, Ministry of Education, School of Resources, Environment and Materials, Guangxi University, Nanning 530000, China; (1) orcid.org/00000002-9013-0612; Email: wangnannan@gxu.edu.cn

\section{Authors}

Ding Chen - College of Chemistry and Chemical Engineering, Guangxi University, Nanning 530000, China

Yu Chen - College of Engineering, Mathematics and Physical Sciences, University of Exeter, Exeter EX4 4PY, U.K.

Kunyapat Thummavichai - College of Engineering, Mathematics and Physical Sciences, University of Exeter, Exeter EX4 4PY, U.K.

Oluwafunmilola Ola - Advanced Materials Group, Faculty of Engineering, The University of Nottingham, Nottingham NG27 2RD, U.K.

Zhiyuan Ma - Guangxi Institute Fullerene Technology (GIFT), Key Laboratory of New Processing Technology for Nonferrous Metals and Materials, Ministry of Education, School of Resources, Environment and Materials, Guangxi University, Nanning 530000, China

Guangsheng Liu - Guangxi Institute Fullerene Technology (GIFT), Key Laboratory of New Processing Technology for Nonferrous Metals and Materials, Ministry of Education, School of Resources, Environment and Materials, Guangxi University, Nanning 530000, China

Yanqiu Zhu - Guangxi Institute Fullerene Technology (GIFT), Key Laboratory of New Processing Technology for Nonferrous Metals and Materials, Ministry of Education, School of Resources, Environment and Materials, Guangxi University, Nanning 530000, China; (o orcid.org/00000002-0866-8852

Complete contact information is available at:

https://pubs.acs.org/10.1021/acsomega.1c05139

\section{Notes}

The authors declare no competing financial interest.

\section{ACKNOWLEDGMENTS}

This work was supported by grants from the National Natural Science Foundation (51972068), Natural Science Foundation of Guangxi (2020JJB160053), and Guangxi Key Laboratory of Manufacturing Systems and Advanced Manufacturing Technology (20-065-40S007). The authors thank Man Mohan Shukla of IIT Kanpur, India, for the reaction mechanism discussion and illustration.

\section{REFERENCES}

(1) Titirici, M. M.; White, R. J.; Brun, N.; Budarin, V. L.; Su, D. S.; del Monte, F.; Clark, J. H.; MacLachlan, M. J. Sustainable carbon materials. Chem. Soc. Rev. 2015, 44, 250-290.

(2) Wang, H.; Feng, H.; Li, J. Graphene and graphene - like layered transition metal dichalcogenides in energy conversion and storage. Small 2014, 10, 2165-2181.

(3) Dai, L.; Chang, D. W.; Baek, J. B.; Lu, W. Carbon nanomaterials for advanced energy conversion and storage. Small 2012, 8, 11301166.

(4) Zhang, H. Ultrathin two-dimensional nanomaterials. ACS Nano 2015, 9, 9451-9469.

(5) Mas-Ballesté, R.; Gómez-Navarro, C.; Gómez-Navarro, J.; Zamora, F. 2D materials: to graphene and beyond. Nanoscale 2011, $3,20-30$.

(6) Liu, J.; Li, W.; Duan, L.; Li, X.; Ji, L.; Geng, Z.; Huang, K.; Lu, L.; Zhou, L.; Liu, Z.; Chen, W.; Liu, W.; Feng, S.; Zhang, Y. A graphene-like oxygenated carbon nitride material for improved cyclelife lithium/sulfur batteries. Nano Lett. 2015, 15, 5137-5142.

(7) Reddy, A. L. M.; Srivastava, A.; Gowda, S. R.; Gullapalli, H.; Dubey, M.; Ajayan, P. M. Synthesis of nitrogen-doped graphene films for lithium battery application. ACS Nano 2010, 4, 6337-6342. 
(8) Lin, Z.; Waller, G. H.; Liu, Y.; Liu, M.; Wong, C. P. 3D Nitrogen-doped graphene prepared by pyrolysis of graphene oxide with polypyrrole for electrocatalysis of oxygen reduction reaction. Nano Energy 2013, 2, 241-248.

(9) Panchakarla, L. S.; Govindaraj, A.; Rao, C. N. R. Boron-and nitrogen-doped carbon nanotubes and graphene. Inorg. Chim. Acta 2010, 363, 4163-4174.

(10) Poshkus, A. C.; Parker, J. A. Studies on nitroaniline-sulfuric acid compositions: Aphrogenic pyrostats. J. Appl. Polym. Sci. 1970, 14, 2049-2064.

(11) Poshkus, A. C. 4-Nitroaniline-2-Sulfonic Acid Preparation and Salt Formation. A New Class of Aphrogens. Prod. R\&D 1971, 10, 72-

(12) Men, Y.; Siebenbürger, M.; Qiu, X.; Antonietti, M.; Yuan, J. Low fractions of ionic liquid or poly (ionic liquid) can activate polysaccharide biomass into shaped, flexible and fire-retardant porous carbons. J. Mater. Chem. A 2013, 1, 11887-11893.

(13) Errede, L. A.; Sinclair, R. A.; Newman, S. Poly (quinoxaline)like chars as room-temperature redox catalysts. React. Polym., Ion Exch., Sorbents 1988, 8, 201-209.

(14) Andreoli, E.; Barron, A. CO2 Adsorption by para-Nitroaniline Sulfuric Acid-Derived Porous Carbon Foam. C 2016, 2, No. 25

(15) Zalba, B.; Marín, J. M.; Cabeza, L. F.; Mehling, H. Review on thermal energy storage with phase change: materials, heat transfer analysis and applications. Appl. Therm. Eng. 2003, 23, 251-283.

(16) Quanying, Y.; Chen, L.; Lin, Z. Experimental study on the thermal storage performance and preparation of paraffin mixtures used in the phase change wall. Sol. Energy Mater Sol. Cells 2008, 92, $1526-1532$.

(17) Velraj, R.; Seeniraj, R. V.; Hafner, B.; Faber, C.; Schwarzer, K. Heat transfer enhancement in a latent heat storage system. Sol. Energy 1999, 65, 171-180.

(18) Tan, L.; Gan, L.; Hu, J.; Zhu, Y.; Han, J. Functional shape memory composite nanofibers with graphene oxide filler. Composites, Part A 2015, 76, 115-123.

(19) Zhang, Q.; Zhao, Y.; Feng, J. Systematic investigation on shape stability of high-efficiency SEBS/paraffin form-stable phase change materials. Sol. Energy Mater. Sol. Cells 2013, 118, 54-60.

(20) Xie, J.; Spallas, J. P. Different contrast mechanisms in SEM imaging of graphene. Agilent Technologies, June 26, 2012.

(21) Stankovich, S.; Dikin, D. A.; Piner, R. D.; Kohlhaas, K. A.; Kleinhammes, A.; Jia, Y.; Wu, Y.; Nguyen, S. T.; Ruoff, R. S. Synthesis of graphene-based nanosheets via chemical reduction of exfoliated graphite oxide. Carbon 2007, 45, 1558-1565.

(22) Li, X.; Cai, W.; An, J.; Kim, S.; Nah, J.; Yang, D.; Piner, R.; Velamakanni, A.; Jung, I.; Tutuc, E.; Banerjee, S. K.; Colombo, L.; Ruoff, R. S. Large-area synthesis of high-quality and uniform graphene films on copper foils. Science 2009, 324, 1312-1314.

(23) Robertson, A. W.; Warner, J. H. Atomic resolution imaging of graphene by transmission electron microscopy. Nanoscale 2013, 5, 4079-4093

(24) Lee, J.; Kim, K.; Park, W. I.; Kim, B. H.; Park, J. H.; Kim, T. H.; Bong, S.; Kim, C. H.; Chae, G.; Jun, M.; Hwang, Y.; Jung, Y. S.; Jeon, $S$. Uniform graphene quantum dots patterned from self-assembled silica nanodots. Nano Lett. 2012, 12, 6078-6083.

(25) Balamurugan, J.; Nguyen, T. T.; Aravindan, V.; Kim, N. H.; Lee, J. H. Flexible Solid-State Asymmetric Supercapacitors Based on Nitrogen-Doped Graphene Encapsulated Ternary Metal-Nitrides with Ultralong Cycle Life. Adv. Funct. Mater. 2018, 28, No. 1804663.

(26) Du, D.; Li, P.; Ouyang, J. Nitrogen-doped reduced graphene oxide prepared by simultaneous thermal reduction and nitrogen doping of graphene oxide in air and its application as an electrocatalyst. ACS Appl. Mater. Interfaces 2015, 7, 26952-26958.

(27) Andreoli, E.; Barron, A. R. Correlating carbon dioxide capture and chemical changes in pyrolyzed polyethylenimine-C60. Energy Fuels 2015, 29, 4479-4487.

(28) Kessel, R.; Schultze, J. W. Surface analytical and photoelectrochemical investigations of conducting polymers. Surf. Interface Anal. 1990, 16, 401-406.
(29) Ejorh, Y. E.; Ilsley, W. H.; Ooi, B. G. Elucidating the Chemisorption Phenomena in SERS Studies via Computational Modeling. Opt. Photonics J. 2018, 8, 212-234.

(30) Ferrari, A. C. Raman spectroscopy of graphene and graphite: disorder, electron-phonon coupling, doping and nonadiabatic effects. Solid State Commun. 2007, 143, 47-57.

(31) Hodkiewicz, J. Characterizing Graphene with Raman Spectroscopy; Thermo Fisher Scientific, 2010; p 51946.

(32) Tallant, D. R.; Friedmann, T. A.; Missert, N. A.; Siegal, M. P.; Sullivan, J. P. Raman spectroscopy of amorphous carbon. MRS Proc. 1997, 498, 37-48.

(33) Kessel, R.; Schultze, J. W. Surface analytical and photoelectrochemical investigations of conducting polymers. Surf. Interface Anal. 1990, 16, 401-406.

(34) Castiglioni, C.; Negri, F.; Rigolio, M.; Zerbi, G. Raman activation in disordered graphites of the $\mathrm{A} 1^{\prime}$ symmetry forbidden $\mathrm{k} \neq$ 0 phonon: the origin of the D line. J. Chem. Phys. 2001, 11 5, 37693778.

(35) Park, S.; An, J.; Potts, J. R.; Velamakanni, A.; Murali, S.; Ruoff, R. S. Hydrazine-reduction of graphite-and graphene oxide. Carbon 2011, 49, 3019-3023.

(36) Geng, D.; Yang, S.; Zhang, Y.; Yang, J.; Liu, J.; Li, R.; Sham, T. K.; Sun, X.; Ye, S.; Knights, S. Nitrogen doping effects on the structure of graphene. Appl. Surf. Sci. 2011, 257, 9193-9198.

(37) Wang, H.; Maiyalagan, T.; Wang, X. Review on recent progress in nitrogen-doped graphene: synthesis, characterization, and its potential applications. ACS Catal. 2012, 2, 781-794.

(38) Takenawa, R.; Komori, Y.; Hayashi, S.; Kawamata, J.; Kuroda, $\mathrm{K}$. Intercalation of nitroanilines into kaolinite and second harmonic generation. Chem. Mater. 2001, 13, 3741-3746.

(39) Manoj, B.; Kunjomana, A. G. Study of stacking structure of amorphous carbon by X-ray diffraction technique. Int. J. Electrochem. Sci. 2012, 7, 3127-3134.

(40) Stankovich, S.; Dikin, D. A.; Piner, R. D.; Kohlhaas, K. A.; Kleinhammes, A.; Jia, Y.; Wu, Y.; Nguyen, S. T.; Ruoff, R. S. Synthesis of graphene-based nanosheets via chemical reduction of exfoliated graphite oxide. Carbon 2007, 45, 1558-1565.

(41) Min, G.; Zengmin, S.; Weidong, C.; Hui, L. Anisotropy of mesophase pitch-derived carbon foams. Carbon 2007, 45, 141-145.

(42) Li, Z. Q.; Lu, C. J.; Xia, Z. P.; Zhou, Y.; Luo, Z. X-ray diffraction patterns of graphite and turbostratic carbon. Carbon 2007, $45,1686-1695$.

(43) Rajan, A. S.; Sampath, S.; Shukla, A. K. An in situ carbongrafted alkaline iron electrode for iron-based accumulators. Energy Environ. Sci. 2014, 7, 1110-1116.

(44) Singh, N.; Ahmad, A. Synthesis and spectrophotometric studies of charge transfer complexes of p-nitroaniline with benzoic acid in different polar solvents. J. Mol. Struct. 2014, 1074, 408-415.

(45) Buzaglo, M.; Bar, I. P.; Varenik, M.; Shunak, L.; Pevzner, S.; Regev, O. Graphite to Graphene: total conversion. Adv. Mater. 2017, 29, No. 1603528

(46) Mittal, G.; Dhand, V.; Rhee, K. Y.; Park, S. J.; Lee, W. R. A review on carbon nanotubes and graphene as fillers in reinforced polymer nanocomposites. J. Ind. Eng. Chem. 2015, 21, 11-25. 